\title{
Análise funcional com enfoque físico de membros superiores em professores com síndrome dolorosa
}

\author{
Emilyn Borba da Silva, Miriam Cabrera Corvelo Delboni, \\ Amara Lúcia Holanda Tavares Battistel, Luis Ulisses Signori
}

Universidade Federal de Santa Maria - UFSM, Santa Maria, RS, Brasil.

\begin{abstract}
Resumo: O labor faz com que o indivíduo se sinta útil, produtivo e valorizado. No entanto, a atividade laboral pode estar associada a lesões devido a condições impróprias para o trabalho, a posturas inadequadas e à realização de movimentos repetitivos. Dentre as principais doenças relacionadas ao trabalho, estão as lesões por esforços repetitivos/doenças osteomusculares relacionadas ao trabalho (LER/DORT). No entanto, as algias são os primeiros sintomas que podem alertar para a prevenção destas lesões. Especificamente no trabalho docente, há uma série de fatores que podem resultar em algias nos membros superiores. Esta pesquisa objetivou identificar a prevalência de algias musculoesqueléticas nos membros superiores em professores, bem como a correlação com a profissão docente e a execução das atividades do cotidiano. Como instrumento, foi utilizada uma entrevista sociodemográfica e o The Arm, Shoulder And Hand (DASH). Esta pesquisa se caracteriza por ser um estudo quantitativo e transversal. Foi utilizado o Teste de Shapiro-Wilk para verificar a normalidade dos dados, e a Correlação de Spearman $(p)$ foi aplicada nos dados de distribuição assimétrica, tendo sido considerada significante a taxa de erro alfa de 5\% $(\mathrm{P}<0,05)$. Os resultados desta pesquisa evidenciam que há alta prevalência de síndrome dolorosa nos membros superiores em professores, associada a diversos fatores da execução do trabalho docente. As algias apresentadas interferiram nas atividades funcionais desses trabalhadores, comprometendo-lhes a qualidade de vida. Assim, entende-se que a prevalência das algias tem significante correlação com o trabalho laboral docente, as quais também acometem a execução das Atividades de Vida Diária (AVDs) e as Atividades Instrumentais de Vida Diária (AIVDs).
\end{abstract}

Palavras-chave: Atividades Cotidianas, Extremidade Superior, Saúde do Trabalhador, Transtornos Traumáticos Cumulativos.

\section{Functional analysis with physical focus on upper limbs in teachers with painful syndrome}

\begin{abstract}
The labor makes the individual to feel useful, productive and valued. However, labor activity may be associated with injuries due to unsuitable work conditions, awkward postures and repetitive movements. The repetitive strain injuries/work-related musculoskeletal disorders (RSI / WMSD) are among the major occupational diseases. Therefore, pain is the first symptom that may alert to prevent these injuries. Teaching has a number of factors that can result in pains in the upper limbs. This research aimed to identify the incidence of musculoskeletal pains in upper limbs in teachers as well as the relationship with the profession and daily activities. A socio-demographic interview and assessment of the Disorders of the Shoulder, Arm and Hand (DASH) were used as instrument for data collection. The research is characterized as a quantitative cross-sectional study. The Shapiro-Wilk test was used to verify the data normality, the Spearman correlation (p) was applied to the data with asymmetric distribution and the rate of alpha error of $5 \%(\mathrm{P}<0.05)$ considered significant. The results show that there is high prevalence of upper limb pain syndrome in teachers, which may be associated with various factors of the teaching job. The presented pains interfered in the functional activities, impaired quality of life and labor. Thus, it is understood that the prevalence of pains have significant correlation due to teaching labor, which also affect the performance of functional daily.
\end{abstract}

Keywords: Daily Activities, Cumulative Trauma Disorders, Occupational Health, Upper Limb. 


\section{Introdução}

A atividade laboral, além de ser uma fonte de subsistência, tem um cunho valorativo, na medida em que possibilita ao sujeito se sentir útil, produtivo e valorizado. $\mathrm{O}$ trabalho é entendido como matriz da integração social e constituidor da identidade do indivíduo (DELIBERATO, 2002; LANCMAN, 2004; CASTEL, 1998).

Com a evolução tecnológica no mundo do trabalho, acreditou-se que haveria uma redução na carga horária, o fim do trabalho penoso, maior tempo para o lazer e, portanto, melhor qualidade de vida. Entretanto, o que pode ser visto é a redução de postos de trabalho e, ao mesmo tempo, uma sobrecarga e a multiplicação das atividades daqueles que permanecem no mercado (LANCMAN, 2004).

Na vida adulta, os indivíduos encontram-se no desenvolvimento pleno de suas capacidades laborais e estão sujeitos a lesôes incapacitantes, que dificultam a realização de atividades significativas para a sua vida. Nesse período, a maioria dos indivíduos encontra-se em uma fase produtiva, realizando-se profissionalmente, inserindo-se no mercado de trabalho, constituindo família e, portanto, vivendo um momento de grandes realizaçôes e de muito empenho pessoal (ANDRADE, 2008).

A maior demanda de trabalho, o excesso de carga horária e a maior exigência do mercado, no entanto, têm levado ao adoecimento dos indivíduos. Isso ocorre, conforme Alves e Rubin (2000), porque a atividade laboral está associada a lesões devidas a condiçóes impróprias para o trabalho, a posturas inadequadas e à realização de movimentos repetitivos.

Dentre os principais transtornos traumáticos cumulativos ligados ao trabalho, estão as lesôes por esforços repetitivos/doenças osteomusculares relacionadas ao trabalho (LER/DORT) (RICE; LUSTER, 2013). No Brasil, os casos de LER/DORT aumentaram de maneira significativa, dando origem a uma das maiores epidemias da vida moderna (OLIVEIRA, 2004; LANCMAN, 2004). Kasch e Nickerson (2005) afirmam que aproximadamente 63\% das 90 mil lesôes por LER nos Estados Unidos afetam o punho, a mão e o ombro.

As lesôes por LER/DORT são tipicamente consideradas transitórias; no entanto, podem resultar em incapacidades permanentes. Elas correspondem a um conjunto de afecçóes relacionadas às atividades laborais. Alguns exemplos incluem danos aos tendóes e às bainhas tendíneas, à lubrificação sinovial dessas bainhas, aos ossos, aos músculos, aos ligamentos, às fáscias, aos vasos sanguíneos, aos nervos, aos cotovelos, aos ombros, ao pescoço, às costas e às pernas (RICE; LUSTER, 2013).

Gasparini, Barreto e Assunção (2005) afirmam que, na última década, o trabalho docente tornou-se foco de vários estudos e pesquisas. Isso ocorreu porque o ritmo acelerado e a sobrecarga de trabalho realizado pelos professores têm gerado grande repercussão na área da saúde pública, já que houve um aumento do adoecimento e do afastamento desses profissionais (SOUZA et al., 2003).

As características do trabalho docente, como esforço repetitivo, ambientes mal planejados ergonomicamente, altas cargas horárias de trabalho em sala de aula e atividades extras realizadas no dia a dia, formam uma rede de fatores que podem resultar em algias nos membros superiores. Tais membros, para Longen (2003), são os locais mais comuns para o aparecimento de LER/DORT. Conforme Carvalho e Alexandre (2006), os professores vêm sendo progressivamente acometidos por diversas patologias, ignorando suas próprias necessidades de saúde, assumindo uma postura conformista e comodista, e não investindo ou não tendo condiçôes para reverter tal quadro.

As ocorrências de algias associadas às lesóes incapacitantes podem dificultar a realizaçáo das atividades cotidianas. Dentre essas atividades, podem-se citar as Atividades de Vida Diária (AVDs) e as Atividades Instrumentais da Vida Diária (AIVDs). Cuidar da higiene pessoal, trabalhar, atender ao telefone, cozinhar e jogar cartas com amigos são alguns exemplos dessas atividades, que podem ter diferentes graus de importância no dia a dia de cada pessoa.

Segundo Foti (2005), as AVDs abrangem as atividades de autocuidado, a mobilidade e a comunicação funcional, e a administraçáo de hardware e de dispositivos ambientais. As AIVDs, por sua vez, incluem a administração doméstica e capacidades para a vida em comunidade. No momento em que essas atividades significativas náo conseguem ser realizadas satisfatoriamente, a qualidade de vida fica, consequentemente, comprometida. Ressalta-se que The WHOQOL Group (1995, p. 1405) definiu qualidade de vida como a

[...] percepção do indivíduo de sua posição na vida, no contexto, da cultura e sistema de valores nos quais ele vive e em relação aos seus objetivos, expectativas, padróes e preocupaçôes. 
Diante disso, é de fundamental importância o enfoque dos profissionais da saúde em fatores geradores de acometimentos, já que isso pode, conforme afirmam Sampaio, Mancini e Fonseca (2002), contribuir para a necessidade da amenização do impacto que determinada condição de saúde traz para a vida pessoal, familiar e social de um indivíduo. Nesse contexto, destaca-se a Terapia Ocupacional, que é uma profissão da área da saúde, cujo objetivo principal é promover e recuperar as condiçôes de indivíduos que apresentam desordens temporárias ou crônicas, para a realização de atividades que sejam significativas em seu cotidiano (CAVALCANTI; GALVĀO, 2007).

Lancman (2004) afirma que diversas pesquisas vêm abordando formas de melhorar a produção, a organização e as condiçóes de trabalho na saúde do trabalhador, mas que são poucas as pesquisas que tratam do conteúdo do trabalho e das relaçóes subjetivas do trabalhador. Assim, esta pesquisa, ao analisar as prevalências de algias musculoesqueléticas no processo laboral de professores e relacioná-las com o cotidiano do indivíduo, visa a trazer contribuiçôes aos estudos da Terapia Ocupacional na área de saúde do trabalhador e na área da reabilitação físico-motora.

Grande parte dos estudos nessa área enfatiza apenas as patologias relacionadas ao trabalho docente, as LER/DORT; no entanto, não abordam os acometimentos relativos à funcionalidade e às dificuldades na execução das atividades do cotidiano. Assim, o que motivou este estudo, além da escassez de trabalhos na literatura referentes a esse tema, foi o impacto causado nas atividades ocupacionais e na qualidade de vida dos professores pela sintomática de algias em membros superiores. O objetivo desta pesquisa consiste, então, em identificar a prevalência de algias musculoesqueléticas que acometem os membros superiores e a correlação das algias com a profissão docente e a execução das atividades do cotidiano de professores de um Colégio Estadual do município de Santa Maria-RS.

\section{Material e métodos}

Esta pesquisa se caracteriza como uma investigação de cunho quantitativo, descritivo e transversal. Gil (1991) afirma que a pesquisa descritiva utiliza técnicas padronizadas de coleta de dados, tais como questionários e instrumentos padronizados de avaliação. A investigação quantitativa atua em níveis de realidade e tem como objetivo trazer à luz dados, indicadores e tendências observáveis (SERAPIONI, 2000).
A pesquisa foi realizada em um Colégio Estadual, do município de Santa Maria- RS. No primeiro contato com o Colégio, foi solicitada a autorização institucional para a realização desta pesquisa.

Foi entrevistada a totalidade de 45 professores que, no momento da pesquisa, atuavam no referido Colégio Estadual de Ensino Fundamental e Médio. Os critérios de exclusão foram: não estar lecionando no Colégio Estadual no qual a pesquisa foi realizada, ter abaixo de 18 anos, não mencionar dor nos membros superiores e não assinar o Termo de Consentimento Livre e Esclarecido. Dos 45 professores entrevistados, 15 foram excluídos por não apresentarem nenhuma sintomatologia álgica nos membros superiores, totalizando 30 professores na amostra da pesquisa.

Os participantes são professores que lecionam no Ensino Fundamental e Médio, e desempenham atividades características do trabalho docente, como, por exemplos, preparação das aulas, uso do quadro negro, correção de provas, atendimento à classe de alunos e utilizaçáo do computador.

Foi utilizado como instrumento para coleta de dados um questionário, que permitiu a coleta de informaçôes sociodemográficas e questóes direcionadas para o objetivo da pesquisa, tais como: idade, sexo, naturalidade, dominância lateral, tempo de profissão docente, carga horária de trabalho diária, atividades realizadas no trabalho e quais atividades além do trabalho docente realiza no dia a dia, realização e frequência de atividade física, presença de dor nos membros superiores, local da dor, se possui diagnóstico médico prévio, tratamento realizado, se relaciona a dor com o trabalho docente, influência da dor nas AVDs, bem como se já recebeu instruçôes de alongamentos, postura e posicionamento correto durante a execução do trabalho.

Utilizou-se, também, o The Arm, Shoulder And Hand (DASH), um instrumento que reflete o impacto funcional de diversas doenças que acometem o membro superior. O DASH é dividido em três partes: a primeira refere-se às AVDs e AIVDs; a segunda diz respeito à atividade de prática esportiva e musical, e a terceira relaciona-se às atividades de trabalho. Nesta pesquisa, foram utilizadas apenas a primeira parte, com 30 questóes, e a terceira parte, com quatro questôes. O DASH é uma avaliação benéfica, pois é específica para o membro superior, podendo ser empregada em pacientes com desordens variadas nesta região, além de permitir comparaçóes entre distintas afecçóes e realidades culturais (BEATON et al., 2005). 
Após a aceitação e a concordância por parte dos professores em participar da pesquisa, tendo assinado o Termo de Consentimento Livre e Esclarecido (TCLE), durante os meses de abril e maio de 2014, foi realizada a aplicação do questionário e do instrumento DASH sob forma de entrevista. Estas ocorreram em seu local de trabalho, com duraçáo aproximada de 30 minutos, para evitar cansaço e condiçóes desfavoráveis que viessem a interferir nos resultados da pesquisa.

Os dados coletados foram analisados e interpretados sob a perspectiva da pesquisa quantitativa. Os valores do DASH foram transformados em um escore de 100, subtraindo 1 e multiplicando por 25: [(Soma das respostas / n $)-1] \times 25$.

Essa transformação é feita para relacionar o DASH a uma escala de 0 a 100 , de modo que, quanto maior é o escore, maior será o grau de disfunção (KENNEDY et al., 2011).

Os dados estão apresentados conforme a média, o desvio padrão (DP), em números absolutos e seus respectivos percentuais. A normalidade dos dados foi verificada pelo Teste de Shapiro-Wilk. A Correlação de Spearman ( $p$ ) foi aplicada nos dados de distribuição assimétrica. A taxa de erro alfa de $5 \%(\mathrm{P}<0,05)$ foi considerada significante.

Esta pesquisa foi orientada e conduzida de acordo com os preceitos éticos preconizados pela Resolução 466/2012 do Ministério da Saúde (BRASIL, 2012), que regulamenta pesquisas que envolvem seres humanos. Foi aprovada e autorizada pelo Comitê de Ética e Pesquisa, da Universidade Federal de Santa Maria (CAAE 26665714.0.0000.5346).

\section{Resultados}

Do total de 45 professores entrevistados, apenas 15 não apresentaram sintomas álgicos e, portanto, foram excluídos da pesquisa por se enquadrarem nos critérios de exclusão. Dentre os 30 professores participantes, dois eram educadores físicos e 28 professores de disciplinas curriculares do Ensino Fundamental, de $1^{\circ}$ ao $9^{\circ}$ ano, e do Ensino Médio, $1^{\circ}$ ao $3^{\circ}$ ano. A Tabela 1 apresenta as características sociodemográficas da amostra, na qual se observa a predominância do sexo feminino $(86,6 \%)$ em relação ao sexo masculino $(13,4 \%)$; as idades variaram entre 24 e 60 anos, sendo a média de 45,6 $( \pm 10,3)$ anos; a dominância lateral foi de destros $(86,6 \%)$ em relação aos sinistros $(13,4 \%)$; o tempo médio de profissão docente foi de $17,3( \pm 10,2)$ anos, e a carga horária semanal de $37,5( \pm 15,0)$ hora/aula.
A Tabela 2 demonstra que a maior prevalência de dor foi localizada no ombro $(73,3 \%)$ e, nas demais articulaçôes do membro superior - cotovelos, punho e dedos-, 26,6\%. A maioria da amostra (90,0\%) relacionou as dores com o trabalho docente, as atividades relatadas que são executadas durante o trabalho docente foram: o uso do quadro negro, a correção de provas, o atendimento à classe de alunos e a utilização do computador. Dos participantes, $56,6 \%$ possuíam diagnóstico médico prévio, mas somente $30,0 \%$ realizaram tratamento. A realização de atividades físicas regulares foi descrita por $56,6 \%$, incluindo caminhada, musculação e dança, e apenas $10,0 \%$ já haviam recebido orientaçóes (alongamentos, postura e posicionamento correto) durante o seu trabalho profissional. A maioria da amostra $(80,0 \%)$ relatou que a dor influencia nas AVDs, dentre as quais prevaleceram atividades do lar, como: varrer a casa, passar pano no chão e carregar as sacolas do mercado.

A correlaçáo dos dados demonstrou que o tempo de docência dos professores está diretamente relacionado com a pontuação do DASH $(p=0,407 ; \mathrm{P}=0,028)$ (Figura 1a). O mesmo aconteceu com o DASH do trabalho e da execução das atividades gerais de vida diária $(p=0,559 ; \mathrm{P}=0,001)$ (Figura $1 \mathrm{~b})$.

Tabela 1. Características sociodemográficas da amostra.

\begin{tabular}{lrr}
\hline \multicolumn{1}{c}{ Variável } & \multicolumn{1}{c}{$\mathbf{n ~ ( \% )}$} & Média \pm DP \\
\hline Sexo feminino (n) & $27(86,6)$ & \\
Sexo masculino (n) & $3(13,4)$ & \\
Idade (anos) & & $45,6 \pm 10,3$ \\
Destro (n) & $27(86,6)$ & \\
Sinistro (n) & $3(13,3)$ & \\
Tempo de docência & & $17,3 \pm 10,2$ \\
(anos) & $37,5 \pm 15,0$ \\
Carga horária & & \\
semanal (horas) & & \\
\hline
\end{tabular}

Valores expressos em n (\%), média \pm DP.

Tabela 2. Características gerais da amostra.

\begin{tabular}{lr}
\hline \multicolumn{1}{c}{ Variável } & \multicolumn{1}{c}{ n (\%) } \\
\hline Dores no ombro (n) & $22(73,3)$ \\
Dores cotovelo, punho e dedos (n) & $8(26,6)$ \\
Relacionam a dor com trabalho (n) & $27(90,0)$ \\
Diagnóstico Médico (n) & $17(56,6)$ \\
Tratamento (n) & $9(30,0)$ \\
Atividades físicas (n) & $17(56,6)$ \\
Receberem instruções (n) & $3(10,0)$ \\
Dor nas AVDs (n) & $24(80,0)$ \\
\hline
\end{tabular}

Valores expressos em n (\%). AVDs: Atividades de vida diária. 

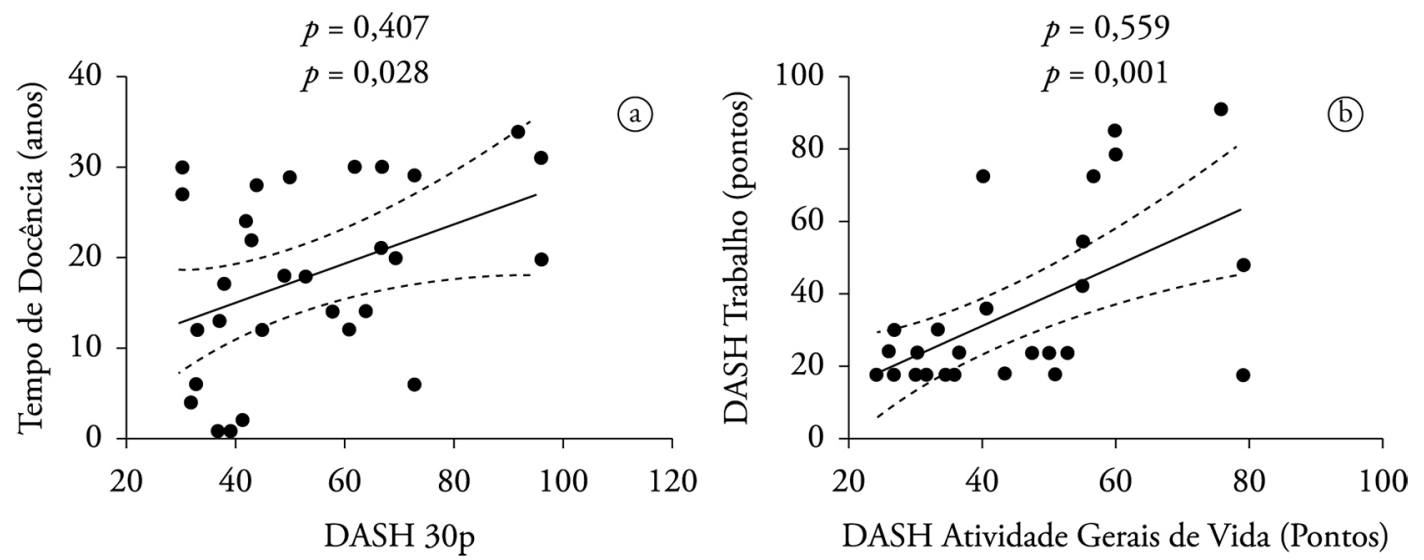

Figura 1. Correlações do tempo de docência e das variáveis do DASH em professores com síndrome dolorosa de membro superior.

\section{Discussão}

Os resultados desta pesquisa evidenciam que há alta prevalência de síndrome dolorosa dos membros superiores em professores, o que pode estar associado a diversos fatores da execução do trabalho dessa classe. Diante disso, ressaltam-se os achados de Dul e Weerdmeester (2004), Miranda, Vera e Pereira (2002), que destacam o fato de a utilização do quadro negro exigir muitas horas de trabalho com o membro superior suspenso e de a pouca movimentação no ambiente de trabalho exigir a execução das atividades na posição estática, podendo ocasionar fadiga.

Porto et al. (2004) citam as síndromes dolorosas relativas às tarefas que exigem força e movimentos repetitivos de dedos, extensão e flexão de punho, elevação do braço acima da altura dos ombros, contração estática dos dedos por tempo prolongado, dentre outros posicionamentos exigidos pelo trabalho docente como fatores preponderantes das algias.

O predomínio do sexo feminino no trabalho docente também foi encontrado em outros estudos (MELO; CAIXETA; CAIXETA, 2010; DUTRA et al., 2005). Essa predominância é justificada no estudo de Delcor et al. (2004) pela presença histórica da mulher no âmbito doméstico, sendo o ato de ensinar considerado uma continuação dessa esfera.

A dor no ombro foi observada em 73,3\% da amostra, prevalência esta que, segundo Coelho et al. (2010), está relacionada à forma como os professores ministram suas aulas. Corroborando esse achado, os estudos de Dutra et al. (2005), Martins e Oesterreich (2013) e Coelho et al. (2010) apontam o ombro como região de maior queixa de algias. Isso se explica: a dor na articulação do ombro é um sintoma bastante comum porque a articulaçáo glenoumeral tem mobilidade considerável, sendo usual o desenvolvimento de alterações degenerativas nessa estrutura (CORRIGAN; MAITLAND, 2000).

Dos professores avaliados, 90,0\% relacionaram as algias com o trabalho docente e relataram ter consciência da correlaçáo entre as exigências dos movimentos decorrentes do processo de trabalho e os males que estes podem ocasionar, principalmente se não executados de maneira correta. Dados semelhantes foram encontrados por Branco et al. (2011): em seus estudos, 79,5\% dos professores entrevistados também acreditam que a sintomatologia dolorosa esteja relacionada ao trabalho.

O baixo índice de professores que realizam tratamento indica que grande parte dessa categoria executa as atividades laborais mesmo com a presença de algias. Sabe-se, entretanto, como afirma Mango et al. (2012), que a dor persistente prejudica a qualidade de vida dos professores.

Um estudo realizado por Coelho et al. (2010) sobre a qualidade de vida de professores e os sintomas osteomusculares revelou que a qualidade de vida foi influenciada negativamente pela Síndrome do Ombro Doloroso (SOD) e que a prevalência de algias no ombro ocorre em mulheres. Mense, Simons e Russel (2008) relacionam o índice de lesão no ombro em mulheres a fatores como a menopausa e a menor massa muscular em membros superiores para dar suporte à articulação do ombro. Tal fato também pode estar associado ao somatório das longas jornadas de trabalho com as AVDs e AIVDs. Quanto a isso, Kraciunas (2007) relata que o acúmulo da jornada de trabalho do professor tende a duplicar devido às atividades realizadas no lar.

Em relação à prática de atividade física, 56,6\% da amostra realiza atividades físicas regularmente, resultado que se assemelha aos de Mango et al. 
(2012), Carvalho e Alexandre (2006), que relacionam essa incidência à falta de tempo, à dupla jornada de trabalho ou a questóes socioeconômicas.

O fato de que apenas $10 \%$ dos professores receberam orientaçóes sobre alongamentos, postura e posicionamento correto durante a execução do seu trabalho profissional mostra a importância do profissional de Terapia Ocupacional nesse campo de atuação. O terapeuta ocupacional pode atuar na análise ergonômica dos instrumentos e mobiliários utilizados no dia a dia dos professores, propor adaptaçóes para o meio ambiente e para os equipamentos, orientar quanto ao uso mais adequado da mecânica corporal e oferecer a utilização de terapêutica específica com a finalidade de combater o aparecimento ou a evolução de sintomas álgicos (OLIVEIRA, 2004; LANCMAN, 2004). Segundo Kraciunas (2007), a melhor maneira de prevenir os distúrbios osteomusculares relacionados ao trabalho é o alongamento da musculatura, a percepção precoce das algias e a conscientização corporal.

De forma semelhante aos dados encontrados no estudo de Melo, Caixeta e Caixeta (2010), 96,3\% dos professores entrevistados afirmaram que a presença da sintomatologia dolorosa impediu a realização das Atividades de Vida Diária. Os sintomas osteomusculares estão, assim, associados ao impedimento da realização das atividades diárias e representam um risco ocupacional para os professores (MANGO et al., 2012; CARVALHO; ALEXANDRE, 2006; MACIEL; FERNANDES; MEDEIROS, 2006; SANTOS; BARRETO, 2001).

Neste estudo, houve uma correlação significante $(p=0,407 ; \mathrm{P}=0,028)$ entre o tempo de docência dos professores e a alta pontuaçáo dos dados do DASH, qual seja: quanto maior o tempo de docência vivenciado pelos professores, maior foi a presença de disfunção em ombro, braço e mão. Esses dados confirmam os resultados encontrados por Cardoso et al. (2009), em que o trabalho laboral docente está associado à maior ocorrência de dores musculoesqueléticas.

A pontuação do DASH geral relacionado às disfunçôes de ombro, braço e mão na execução das atividades gerais de vida diária também se correlacionou ao DASH referente ao trabalho $(\mathrm{p}=0,559 ; \mathrm{P}=0,001)$. Os dados apresentados indicam proporcionalidade significativa na pontuação das duas avaliaçôes, o que significa que, ao ocorrer uma disfunção que prejudique a realização das AVDs e AIVDs, haverá um impacto na execução da atividade laboral. Corroborando essa ideia, Melo, Caixeta e Caixeta (2010) afirmam que o aumento das incapacidades ocupacionais prejudica o desempenho dos professores na execução de seu trabalho, resultando na diminuição de sua produtividade.

\section{Conclusão}

Os professores entrevistados nesta pesquisa apresentaram elevada prevalência de algias nos membros superiores, especialmente na regiáo do ombro. Os fatores que provavelmente levam a isso são ligados ao gênero, à falta de exercícios para aumentar a resistência, à fadiga da musculatura dos membros superiores pela permanência por tempo prolongado e à repetiçáo de movimento de escrita no quadro/lousa com a flexão e abdução do ombro acima de $90^{\circ}$.

Portanto, pôde-se observar que a prevalência das algias tem significante correlação com o trabalho laboral docente, as quais também acometem a execução das AVDs e AIVDs, e, consequentemente, a qualidade de vida desses professores. Tais resultados são relevantes para a classe docente, alertando-a sobre as prevençóes quanto a desenvolver LER/DORT e para a discussão de possíveis medidas de prevenção e tratamento com profissionais especializados.

Sugere-se a realização de novas pesquisas, principalmente do tipo qualitativo, em que sejam incluídos outros dados sobre sofrimento e sobrecarga psíquica dos professores que atuam em escolas públicas e privadas no contexto brasileiro, avaliando como isso ocorre nas diversas regióes do país. Acredita-se que esses fatores possivelmente influenciem o aumento da prevalência de algias dos membros superiores em professores.

\section{Referências}

ALVES, D. S.; RUBIN, M. R. Os sintomas dolorosos relacionados à atividade ocupacional do fisioterapeuta. Fisio \& Terapia, Rio de Janeiro, v. 4, n. 20, p. 21-22, 2000.

ANDRADE, M. O. O impacto das sequelas motoras decorrentes do Acidente Vascular Cerebral no indivíduo produtivo e suas repercussöes na família. 2008. 137 f. Dissertação (Mestrado em Psicologia Clínica) - Universidade Católica de Pernambuco, Recife, 2008.

BEATON, D. E. et al. Development of the QUICKDASH: comparison of three item-reduction approaches. The Journal of Bone and Joint Surgery, Boston, v. 87, n. 5, p. 1038-1046, 2005.

BRANCO, J. C. et al. Prevalência de sintomas osteomusculares em professores de escolas públicas e privadas do ensino fundamental. Fisioterapia em Movimento, Curitiba, v. 4, n. 2, p. 307-314, 2011.

BRASIL. Resolução no 466, de 12 de dezembro de 2012. Diário Oficial [da] República Federativa do Brasil, Brasília, DF, 13 jun. 2012. Seção 1. p. 59. Disponível em: <http://www.conselho.saude.gov.br/resolucoes/2012/ Reso466.pdf>. Acesso em: 29 jun. 2014. 
CARDOSO, J. P. et al. Prevalência de dor musculoesquelética em professores. Revista Brasileira de Epidemiologia, Sáo Paulo, v. 12, n. 4, p. 1-10, 2009.

CARVALHO, A. J. F. P.; ALEXANDRE, N. M. C. Sintomas osteomusculares em professores do ensino fundamental. Revista Brasileira de Fisioterapia, São Paulo, v. 10, n. 1, p. 35-41, 2006.

CASTEL, R. As metamorfoses da questão social: uma crônica do salário. Petrópolis: Vozes, 1998.

CAVALCANTI, A.; GALVÃO, C. Terapia Ocupacional: fundamentação e prática. Rio de Janeiro: Guanabara Koogan, 2007.

COELHO, C. T. et al. Prevalência da síndrome do ombro doloroso (SOD) e sua influência na qualidade de vida em professores de uma instituição privada de nível superior na cidade de Lauro de Freitas, Bahia. Revista Baiana de Saúde Pública, Salvador, v. 34, p. 19-29, 2010. Suplemento 1 .

CORRIGAN, B.; MAITLAND, G. B. Prática clínica, ortopedia \& reumatologia: diagnóstico e tratamento. São Paulo: Premier, 2000.

DELCOR, N. S. et al. Condiçóes de trabalho e saúde dos professores da rede particular de ensino de Vitória da Conquista, Bahia, Brasil. Cadernos de Saúde Pública, Rio de Janeiro, v. 20, n. 1, p. 187-196, 2004.

DELIBERATO, P. C. P. Fisioterapia preventiva: fundamentos e aplicaçóes. São Paulo: Manole, 2002.

DUL, J.; WEERDMEESTER, B. Ergonomia prática. São Paulo: Edgard Blücher, 2004.

DUTRA, D. et al. Prevalência de algias nos ombros em professores da rede municipal de ensino fundamental de Umuarama - PR no ano de 2004. Arquivos de Ciências da Saúde da UNIPAR, Umuarama, v. 9, n. 2, p. 79-84, 2005.

FOTI, D. Atividades da Vida Diária. In: PEDRETTI, L. W.; EARLY, M. B. Terapia Ocupacional: capacidades práticas para disfunçôes físicas. São Paulo: Roca, 2005. p. 132-183.

GASPARINI, S. M.; BARRETO, S. M.; ASSUNÇÃO, A. A. O professor, as condições de trabalho e os efeitos sobre sua saúde. Educação e Pesquisa, São Paulo, v. 31, n. 2, p. 189-199, 2005.

GIL, A. C. Como elaborar projetos de pesquisa. São Paulo: Atlas, 1991.

KASCH, M. C.; NICKERSON, E. D. Lesões nas Mãos e nos Membros Superiores. In: PEDRETTI, L. W.; EARLY, M. B. Terapia Ocupacional: capacidades práticas para disfunçôes físicas. São Paulo: Roca, 2005. p. 875910.

KENNEDY, C. A. et al. Deficiência do braço, ombro e mão (DASH): o DASH e manual do usuário quick DASH outcome measure. Toronto: Institute for Work \& Health, 2011.
KRACIUNAS, W. Saúde: corpo movimento e saúde. Revista do Professor-SINPRO Sindicato dos professores do $A B C$, São Paulo, n. 7, p. 32-33, 2007.

LANCMAN, S. Construção de Novas Teorias e Práticas em Terapia Ocupacional, Saúde e Trabalho. In: LANCMAN, S. Saúde, trabalho e Terapia Ocupacional. São Paulo: Roca, 2004. p. 71-83.

LONGEN, W. C. Ginástica laboral na prevenção de LER/ DORT? Um estudo reflexivo em uma linha de produção. 2003. 130 f. Dissertação (Mestrado em Engenharia de Produção) - Universidade Federal de Santa Catarina, Florianópolis, 2003.

MACIEL, A. C. C.; FERNANDES, M. B.; MEDEIROS, L. S. Prevalência e fatores associados à sintomatologia dolorosa entre profissionais da indústria têxtil. Revista Brasileira de Epidemiologia, São Paulo, v. 9, n. 1, p. 94-102, 2006. http://dx.doi.org/10.1590/S1415$-790 \times 2006000100012$.

MANGO, M. S. M. et al. Análise dos sintomas osteomusculares de professores do ensino fundamental em Matinhos (PR). Fisioterapia em Movimento, Curitiba, v. 25, n. 4, p. 785-794, 2012

MARTINS, S. M. F. C.; OESTERREICH, S. A. Verificação da incidência de queixas de incômodos osteomusculares em Professores da rede estadual de ensino da cidade de Palmas-PR. FIEP Bulletin On-line, Foz do Iguaçu, v. 83, p. 1-7, 2013.

MELO, E. M. N.; CAIXETA, G. F; CAIXETA, A. Prevalência de lesôes osteomusculares em professores do ensino fundamental. Revista Eletrônica Saúde CESUC, Cataláo, n. 1, p. 1-13, 2010.

MENSE, S.; SIMONS, D. G.; RUSSEL, I. J. Dor muscular, natureza, diagnóstico e tratamento. São Paulo: Manole, 2008.

MIRANDA, T. E. C.; VERA, R. P.; PEREIRA, E. R. Equipamento de apoio para membros superior: uma nova proposta ergonômica. Revista Brasileira de Odontologia, Rio de Janeiro, v. 59, n. 5, p. 338-340, 2002.

OLIVEIRA, A. J. Terapia Ocupacional: perspectiva para a educação em saúde do trabalhador. In: LANCMAN, S. Saude, trabalho e Terapia Ocupacional. São Paulo: Roca, 2004. p. 1-17.

PORTO, L. A. et al. Doenças ocupacionais em professores atendidos pelo centro de estudos da saúde do trabalhador (CESAT). Revista Baiana de Saúde Pública, Salvador, v. 28, n. 1, p. 33-49, 2004.

RICE. V. J.; LUSTER, S. Restaurando a Competência para o papel de Trabalhador. In: RADOMSKI, M. V.; LATHAM, C. A. T. Terapia Ocupacional para disfunçōes físicas. São Paulo: Santos, 2013. p. 255-281.

SAMPAIO, R. F.; MANCINI, M. C.; FONSECA, S. T. Produção científica e atuação profissional: aspectos que limitam essa integração na fisioterapia e na terapia ocu- 
pacional. Revista Brasileira de Fisioterapia, São Carlos, v. 6, n. 3, p. 113-118, 2002.

SANTOS, F. S.; BARRETO, S. Atividade ocupacional e prevalência de dor osteomuscular em cirurgióes dentistas de Belo Horizonte, Minas Gerais, Brasil: contribuição ao debate sobre os distúrbios osteomusculares relacionados ao trabalho. Cadernos de Saúde Pública, Rio de Janeiro, v. 7, n. 1, p. 181-193, 2001.

SERAPIONI, M. Métodos qualitativos e quantitativos na pesquisa social em saúde: algumas estratégias para a integração. Ciência \& Saúde Coletiva, Rio de Janeiro, v.
5, n. 1, p. 187-192, 2000. http://dx.doi.org/10.1590/ S1413-81232000000100016.

SOUZA, K. R. et al. A trajetória do Sindicato Estadual dos Profissionais da Educação do Rio de Janeiro (Sepe-RJ) na luta pela saúde no trabalho. Ciência \& Saúde Coletiva, Rio de Janeiro, v. 8, n. 4, p. 1057-1068, 2003.

THE WHOQOL GROUP - TWG. The World Health Organization quality of life assessment (WHOQOL): position paper from the World Health Organization. Social Science and Medicine, New York, v. 41, n. 10, p. 1403-1409, 1995. PMid:8560308.

\section{Contribuição dos Autores}

Emilyn Borba da Silva: pesquisadora, responsável por coleta, redação e elaboração da pesquisa e do presente artigo. Miriam Cabrera Corvelo Delboni e Amara Lúcia Holanda Tavares Battistel: revisoras do artigo e concepção do texto. Luis Ulisses Signori: responsável pela análise dos dados e revisor dos resultados. Todos os autores aprovaram a versão final do artigo. 